\title{
Diseños curriculares e inteligencia social en zonas urbanas y rurales de Colombia
}

\section{Curriculum designs and social intelligence in Colombian urban and rural areas}

DOI: http://dx.doi.org/10.17981/cultedusoc.12.1.2021.17

Recibido: 18 de mayo de 2020 Aceptado: 14 de agosto de 2020 Publicado: 31 de diciembre de 2020

\author{
Juan Camilo Lotero-Franco \\ Universidad Católica Luis Amigó. Medellín (Colombia) \\ Juan.loterofr@amigo.edu.co \\ Yusty Carolina Restrepo-Segura (1) \\ Universidad Católica Luis Amigó. Medellín (Colombia) \\ carolinasegura2000@gmail.com \\ Leisy Magdali Arroyave-Taborda \\ Universidad Católica Luis Amigó. Medellín (Colombia) \\ leisymag3@gmail.com
}

Para citar este artículo:

Lotero-Franco, J., Restrepo-Segura, Y. y Arroyave-Taborda, L. (2021). Diseños curriculares e inteligencia social en zonas urbanas y rurales de Colombia. Cultura, Educación y Sociedad, 12(1), 255-274. DOI: http://dx.doi.org/10.17981/cultedusoc.12.1.2021.17

\section{Resumen}

El presente artículo de revisión tiene como objetivo develar la importancia de integrar la inteligencia social en los diseños curriculares y sus aportes al fortalecimiento de habilidades prosociales, considerando las características y necesidades propias de zonas rurales y urbanas de Colombia. Se plantea una revisión sistemática, desde el paradigma histórico hermenéutico, enfoque cualitativo; como estrategia de investigación se utilizó la revisión documental que incluye artículos de revisión y de investigación, artículos de reflexión, tesis posgraduales y libros teóricos de los últimos 10 años, presentes en bases de datos como: Digital Magisterio; Digitalia; EBSCO host DATA BASES; ScienceDirect; Scopus. Las categorías de análisis fueron: educación rural; diseño curricular; cognición e inteligencia social. Los principales resultados fueron agrupados en tres categorías de análisis: importancia de la inteligencia social en los escenarios educativos; diferencias entre los sectores educativos rurales y urbanos: repercusiones en el desarrollo de la inteligencia social; e inclusión de la inteligencia social en los diseños curriculares. Como conclusión resalta que el currículo más allá de brindar una formación académica debe dar respuesta al desarrollo integral y personal de los estudiantes que se forman. De esta manera toma importancia el desarrollo de la Inteligencia social en el entorno educativo, apuntando a las particularidades que se presentan en los procesos de aprendizaje individual y colectivo.

Palabras clave: Interacción social; aprendizaje social; escuela rural; escuela urbana; educación básica

\begin{abstract}
The present review article aims to reveal the importance of integrating social intelligence in curricular designs and its contributions to strengthening prosocial skills, considering the characteristics and needs of rural and urban areas of Colombia. A systematic review is proposed, from the hermeneutical historical paradigm, qualitative approach; As a research strategy, the documentary review was used, which includes review and research articles, reflection articles, postgraduate theses and theoretical books from the last 10 years, present in databases such as: Digital Teaching; Digitalia; EBSCO host DATA BASES; ScienceDirect; Scopus. The categories of analysis were: rural education; Curricular design; cognition and social intelligence. The main results were grouped into three categories of analysis: importance of social intelligence in educational settings; differences between rural and urban educational sectors: repercussions on the development of social intelligence; and inclusion of social intelligence in curricular designs. In conclusion, it highlights that the curriculum, beyond providing an academic training, must respond to the integral and personal development of the students who are trained. In this way, the development of Social Intelligence in the educational environment becomes important, pointing to the particularities that arise in the individual and collective learning processes.
\end{abstract}

Keywords: Social interaction; social learning; rural school; urban school; basic education 


\section{INTRODUCCIÓN}

El desarrollo de los procesos biológicos, físicos y psicoafectivos en los seres humanos, aportan significativamente a la adquisición de las habilidades socioculturales (Papalia y Martorell, 2017). De esta manera, el proceso de reconocimiento emocional, el procesamiento cognitivo para la toma de decisiones, la utilización del lenguaje para la interacción entre los individuos, e incluso la construcción de la personalidad resultan fundamentales para los procesos de socialización saludable, tal como lo plantea el Ministerio de Salud Nacional de Colombia (MinSalud, 2012). A nivel biológico y físico, la potencial maduración de la corteza prefrontal en el cerebro, se da a partir de los 5-6 años de edad, que permite configurar los procesos psicológicos básicos como la percepción, la atención y la memoria. Así como los superiores: el pensamiento, el lenguaje — verbal y no verbal- y la metacognición (León y Cárdenas, 2016). Desde el plano psicoafectivo, este proceso de maduración, potencia la capacidad de reconocer las propias emociones y las que de otros (Calle, 2014).

Los espacios de crecimiento y socialización de niños y niñas han de estar ambientados apropiadamente por el Estado, la familia y la sociedad, para favorecer el desarrollo de las funciones cognitivas básicas y superiores (Guzmán, Fernández, y Villalba, 2016). Las acciones que se tomen durante este periodo corto e irrepetible en la vida de todo sujeto, tendrán consecuencias directas en el desarrollo pleno de las potencialidades humanas o por el contrario, en la afectación del bienestar, salud y desarrollo de individuos capaces de interactuar en sociedad, en otras palabras, obstaculizan la posibilidad del desarrollo de la cognición y la inteligencia social (Tirapú-Ustárroz, Pérez-Sayes, Erekatxo-Bilbao y Pelegrín-Valero, 2007; Guzmán et al., 2016). Esto podría llevar a pensar que, considerar un individuo aislado de su grupo social, lo pondría en cierta desventaja en cuanto a su supervivencia, dado que el cerebro humano está diseñado para conectar —no sólo biológica, sino psicoafectivamente - con las experiencias de otros (Bassett \& Gazzaniga, 2011).

Goleman (2006) ha desarrollado en su libro inteligencia social, la nueva ciencia para mejorar las relaciones humanas, el concepto de inteligencia social. Lo sustenta desde los planteamientos de la teoría de la mente y las "neuronas espejo" (Rizzolatti, Fadiga, Gallese \& Fogassi, 1996), así como desde una perspectiva psico-social. Así, el autor expone que el funcionamiento de la amígdala cerebral y con el apoyo de la vía inferior cerebral — de procesamiento emocional automático — junto con la vía superior — de procesamiento emocional controlado- permitirán que los sujetos conozcan la forma en que funcionan las relaciones sociales y al mismo tiempo, elaboren las estrategias correctas, para comportarse adecuadamente en ellas. Estas relaciones sociales no solo irán modificando la forma, el tamaño y el número de neuronas de cada sujeto, sino que irán influyendo en su carácter e incluso en su salud física y mental. Esta afirmación va en línea con lo propuesto por Écija y Velasco (2014) en la que la inteligencia emocional no solo se considera en la perspectiva del "yo" y "mis emociones" sino en la del "nosotros" y "nuestras emociones".

De este modo, el desarrollo psicoafectivo individual y social puede verse mediado en escenarios de interacción social continuo, tales como la familia, la escuela y las demás instituciones de socialización, a las que se enfrenta el ser humano a lo largo de su existencia. En el ámbito educativo en Colombia existe, por ejemplo, un marco normativo a través de la Ley 115 (1994), junto con la Ley de Convivencia Escolar (Ley 1620, 2013) y su Decreto 
Reglamentario 1965 (2013) en el que se brindas las disposiciones para que todas las instituciones educativas promuevan una formación para la justicia, la paz, la solidaridad, la confraternidad, el cooperativismo y la formación de los valores humanos; lo que autores como Rodelo y Castro (2016) definen como educabilidad para el desarrollo humano. Sin embargo, las estrategias para resolver diversas problemáticas cotidianas siguen tramitándose violentamente, especialmente en los contextos rurales donde existen "tensiones sociales permanentes, acompañado de violencia agenciada por actores diversos desde el Estado, los lati-fundistas, la insurgencia, la contrainsurgencia y los narcotraficantes" (López, 2018, p. 146).

En Colombia, contextos tanto urbanos como rurales, existen diversos factores de riesgo psicosocial, entre ellos el alto índice de desescolarización, el flagelo de los diferentes tipos de violencia a nivel individual, social y cultural, el ámbito educativo queda en un lugar de vulnerabilidad, dadas las desventajas en relación con la ciudad: la falta de vías de acceso; los escasos materiales didácticos; déficit de acceso tecnológico; la poca preparación de los docentes, ligado a bajos niveles de rendimiento académico, son factores que favorecen la participación de niños y jóvenes en la fuerza laboral y aumenta la deserción escolar, principalmente, en la educación media (Figueroa-Torres, Pérez-Bello, Villamarín-Monroy y Rincón-Pérez, 2018). Sin considerar otros factores, como los de orden público, que impactan negativamente la cobertura y la calidad de la educación, convirtiéndose en un ciclo repetitivo de la pobreza integral y limitando el desarrollo de la cognición, la interacción social y el aprendizaje de resolución de diversos conflictos cotidianos sin usar la violencia. Una problemática que ocurre, no sólo a nivel nacional sino a nivel suramericano, como logra evidenciarse a lo largo de los textos expuestos por Figueroa et al. (2018), Corvalán (2006) e Hincapie, Montoya y Bustamante (2017).

Con lo expuesto hasta ahora, se intenta, además, poner en evidencia la importancia que adquiere el rol del docente de los centros educativos, precisamente en la mediación en el desarrollo de las habilidades prosociales - agrupadas en el concepto de la inteligencia social - y una de las herramientas que podrán ser útiles para dicho fin. La psicología, la pedagogía, la neurociencia social y cognitiva, y la psicología social han procurado explicar diferentes fenómenos en relación con la educación (Écija y Velasco, 2014), poniéndola en el lugar de generadora de diversos desafíos a nivel cognitivo, para resolver situaciones con diversos objetos de la realidad que involucran las matemáticas, física, química, etc., y que se desarrollan constantemente. Pero para que la educación en estas competencias se complete, deben suceder diversas interacciones sociales entre sujetos que enseñan y otros que aprenden y que forman un entramado social, paralelo al objetivo de la enseñanzaaprendizaje. Cada una de las historias particulares de estos actores del proceso educativo, confluyen y engranan diversos escenarios geográficos, ideológicos, políticos, culturales y económicos. Dicho engranaje da soporte a los contenidos formativos en la escuela, así lo propone Romero (2011), cuando habla de currículo formal (plan de estudios); currículo real (puesta en práctica); y currículo oculto (tensiones entre el currículo formal y el real, por la relación docente-alumno) (Ratto, 2015).

Si bien existen unos contenidos señalados en el currículo formal, posiblemente su, desarrollo en el plano real, no esté siendo completamente explícito; incluso, en aquel currículo oculto que tiene lugar en la cotidiana y constante relación docente-estudiante, no logran 
evidenciarse las estrategias para que la inteligencia social sea desarrollada y con ella, la construcción del sufrimiento moral; establecimiento de redes de apoyo; dinámicas familiares diversas; y resolución de conflictos por vías dialógicas (Espejo, 2016; Ratto, 2015; Ruíz-Ruíz, García-Ferrer \& Fuentes-Durá, 2006; López, 2018), consolidando el papel de la educación como instancia fundamental para el "cambio de las realidades cercanas" (Mejía, 2011, p. 34).

Así, surge el interés de una visión que apunta al desarrollo de la inteligencia social desde las propuestas de diseños curriculares y sus aportes al fortalecimiento de habilidades de interacción, relaciones sociales sanas, y aprendizaje de estrategias y mecanismos pacíficos para la resolución de diferentes problemas en contextos específicos, por medio del análisis del sistema educativo actual que, parece estar respondiendo a un sistema estandarizado de comparación del desempeño de estudiantes y docentes con los de otros países, a partir del desempeño en las materias básicas - matemáticas, ciencias naturales, lenguaje, ciencias sociales e inglés - aislando los procesos de educación y desarrollo del área socioafectiva (Preal, 1998, p. 15), situaciones que continúan generando más contenidos de enseñanza desde esta perspectiva y estableciendo estándares o niveles educativos que históricamente, no han sido alcanzables en sectores aislados, en los ámbitos urbanos y especialmente, en los rurales (Corvalán 2006; Cossio, 2014; Rivas 2015; Ratto 2015).

\section{Metodología}

La investigación se orientó desde un paradigma histórico hermenéutico, a partir del cual es posible la comprensión de los textos y que según Creswell (1998), permite reflexionar sobre las interpretaciones consultadas de los diferentes autores citados, con la intención de lograr la comprensión del constructo inteligencia s social en los diseños curriculares y su importancia en el ámbito urbano y rural en Colombia. Se orientó desde un enfoque cualitativo, con estrategia de revisión documental, entendida para el caso como un proceso de revisión mediante el cual se buscó de manera específica develar reflexivamente el conocimiento acumulado sobre la temática mencionada (Vélez y Galeano, 2002).

De esta manera, se indago sobre la información disponible en el tema, por medio de un rastreo, recolección, organización, sistematización y análisis de un conjunto de documentos, entre artículos y libros sobre los temas que involucran el desarrollo de la inteligencia social, desde las propuestas curriculares en las zonas urbanas y rurales de Colombia. La información obtenida fue consolidada en una matriz de análisis que permitió discriminar la información según las categorías, el tipo de documento, el año de publicación, los resultados obtenidos y las conclusiones ofrecidas.

Para la búsqueda inicial, se determinaron como categorías de análisis: "educación rural", "diseño curricular", "cognición social", "inteligencia social", "ruralidad en Colombia" "urbanidad en Colombia". Estos constructos se integraron en formas diversas, rastreando información a nivel local, nacional e internacional, tanto en español y principalmente en inglés y portugués. En primera instancia, se hizo búsqueda de artículos y libros que hubiesen sido publicados en los últimos cinco años. Sin embargo, la información encontrada no satisfacía el objetivo inicial de la búsqueda, por lo que fue necesario expandir el rastreo a los documentos publicados en los últimos 10 años. De igual forma, se decidió incluir libros de base que, aunque de data lejana, pudiera dar soporte a las unidades de análisis. 
Para la recolección de la información, la búsqueda documental tuvo lugar entre octubre del 2018 y abril del 2019 en bases de datos como Digital Magisterio, Digitalia, EBSCO host DATA BASES, ScienceDirect, Scopus. El principal criterio de inclusión de los textos radicó en que los mismos apuntaran a resolver inquietudes en el ámbito educativo colombiano, desde la perspectiva de la inteligencia emocional y/o social. También se incluyeron aquellos que problematizaran o apuntaran a entender las diferencias de la educación rural y urbana en Colombia. Se revisaron artículos de investigación original — de corte cuantitativo y cualitativo-, artículos de revisión descriptiva, artículos de reflexión, tesis ubicadas en repositorios y libros teóricos. De manera que, se descartaron aquellos documentos que no abordaran los núcleos temáticos.

En este sentido, se posibilitó entender cómo se ha abordado el concepto de la inteligencia social, desde las perspectivas neuropsicológicas y psico-sociales, en entornos educativos rurales y urbanos. De forma tal, como dicen Hernández, Fernández y Baptista (2014), se diera lugar a la categorización de la información, para mayor comprensión del fenómeno investigado. En este caso fueron entre los sectores educativos rurales y urbanos: repercusiones en el desarrollo de la inteligencia social; e inclusión de la inteligencia social en los diseños curriculares.

\section{REsultados y Discusión}

A continuación, se relacionan antecedentes importantes como rastreos bibliográficos, estados del arte y resultados de investigaciones que, permitieron generar 3 apartados en correspondencia con: la importancia de considerar el desarrollo de la Inteligencia Social al interior de los escenarios educativos (IS); las diferencias entre los sectores educativos rurales y urbanos en Colombia, aunado a las implicaciones que estas tienen en el desarrollo de la IS, y la importancia de incluirla de forma explícita en los escenarios educativos a través de los diseños curriculares.

\section{Importancia de la Inteligencia social en los escenarios educativos}

Las escuelas deben considerar aquellas características que pueden llegar a ser universales como la inteligencia cognitiva, para potenciarla en los estudiantes, pero también deben asumir un papel activo con aquellas variaciones en el entendimiento del entorno por medio de las relaciones interpersonales, su evaluación y consolidación, apoyado en lo que dice Ardila (2011) y Villamizar y Donoso (2013), el esfuerzo aumentaría, considerando que, aquellas variaciones son las que diferencian según etnias, desarrollo cultural, grupos etarios, incluso, las divergencias individuales a nivel de habilidades, motivaciones e intereses, es decir, no bastaría con pensarse un modelo único, estandarizado o universal, para fomentar el desarrollo de las habilidades sociales.

Así lo menciona Sacristán (2001), al hablar de la capacidad de sociabilidad, como la raíz profunda de pertenencia a un momento y espacio específico en el mundo, que aporta significados y configura el comportamiento de quienes están compartiendo el mismo contexto; de este modo, es fundamental que en los escenarios educativos se generen contextos, a partir de las particularidades de los estudiantes y que sean emocionalmente seguros, 
para que la interacción que se haga explícita, garantice el desarrollo de individuos atentos, productivos, saludables mentalmente, y con un alto potencial para el éxito en lo que realice en sociedad, tal como lo afirman Koplow (2005) y Albrecht (2006).

Trabajos investigativos, como los desarrollados por Écija y Velasco (2014), Sternberg et al. (2000), Bar-On, Tranel, Denburg y Bechara (2003), se han preocupado por entender cómo la afectividad ha influenciado en las relaciones sociales, y cómo el uso de las emociones favorecería los procesos de enseñanza y aprendizaje. La perspectiva psicosocial, lo ha abordado desde el concepto de las representaciones sociales de Moscovici, y aunque poco se han articulado con la explicación dada por las bases neurobiológicas, las neurociencias sociales han estado introduciendo la relación entre el sentir y el pensar, entre la cognición y la emoción, dejando claro que, las personas se vinculan constantemente, valiéndose de sus emociones. El cerebro opera como una máquina que puede predecir las conductas, los pensamientos y las intenciones de los otros. A esta capacidad se le ha denominado de varias formas, entre ellas, teoría de la mente, cognición social o mentalización, y explican que existen redes neurales (neuronas espejo) que se especializan en crear hipótesis sobre cómo opera la mente de las demás personas, estableciendo conexiones entre las propias experiencias sensitivas y emocionales con las de los demás, posibilitando anticiparse con acierto a sus conductas.

Dirían Sternberg et al. (2000) que, para entender el entorno circundante, por un lado, estaría la inteligencia práctica, como factor cognitivo de base para las conductas medibles y observables tanto en humanos como en animales, y por otro lado, las habilidades socio-emocionales para identificar las situaciones sociales y la forma correcta de gestionar emociones y sentimientos propios y de los demás. Si bien es necesario implementar recursos cognitivos para lograr entender las propias emociones, se requieren otro tipo de esfuerzos para dimensionar las situaciones circundantes y contextualizar las inferencias que se realizan y así, generar respuestas socialmente precisas, haciendo que se vaya más allá de lo individual, para mediar con las ofertas y demandas socio-culturales situacionales. Lo anterior, se soporta además, en la unificación de las ideas propuestas por Tikka, (2006); Weis y Conzelmann (2015) y Sacco, Merold, Lui, Lustgraaf, y Barry (2016), para llegar a la idea de que, a la hora de ser más exactos en las percepciones sociales, la inteligencia social juega un papel más importante que la inteligencia emocional, dada su asociación con el procesamiento de la información social, que va más allá del reconocimiento de expresiones faciales de la emoción, sino que implica el reconocimiento y detección de señales sociales (Goleman, 2006).

Es decir, en palabras de Ardila (2011), la inteligencia práctica o cognitiva, se trata de una habilidad general, de las que se desprenden otras específicas, como habilidades y talentos puntuales. De este modo, alguien con altos niveles de inteligencia, está en la capacidad de adaptarse, de un mejor modo, a su ambiente físico y social. De igual forma, podrá elegir los espacios en que pueda desempeñarse mejor, incluso, con la potencialidad de cambiarlo y lograr encajar de mejor modo y solucionando situaciones nuevas o inesperadas y manteniéndose intacto. Sin embargo, Manes y Niro (2014) proponen de manera adicional que, los recursos a nivel cognitivo requeridos para resolver problemas con objetos (no humanos), difieren de aquellos que se necesitan para identificar los signos y estímulos sociales (como reconocer al otro y su intención hacia los otros), implicando una memoria de 
tipo emocional, que permite reconocer al amigo y al que no lo es y entender las emociones que permitan interactuar con cada uno de estos roles, basados en la experiencia diaria.

Maree (2001) afirma, por ejemplo, que mientras que los saberes y aptitudes cognitivos, reflejados en altos coeficientes intelectuales, pueden ser la base del logro de buenas calificaciones por parte de los estudiantes en las escuelas, la inteligencia social, podría convertirse en un predictor del éxito de los mismos en los escenarios profesionales. También afirma que, el rendimiento escolar, la aptitud y el Coeficiente Intelectual (CI) son predictores en un 9\% en la consecución de un lugar de trabajo, frente a un 36\% y 40\% que ofrece el desarrollo de la inteligencia socio-emocional.

Lo anterior, pone en el plano consciente, la importancia y necesidad de educar para la Inteligencia social, haciendo aportes fundamentales en la construcción de la cultura escolar, fomentando el desarrollo de ciudadanos competentes, capaces de compartir espacios con otros, de manera pacífica y constructiva — no de una forma utópica y permanentemente armoniosa - considerando que los conflictos que ocurran en el entorno, puedan ser abordados con mínimos niveles de agresividad y en la búsqueda constante de favorecer la dignidad de todos los actores involucrados. Es por esto que, Soon, Brass, Heinze y Haynes (2008) proponen que, el desarrollo de la inteligencia social, debería darse de forma explícita y con contenidos específicos, basados en algunos principios que la fundamentan: el reconocimiento de la humanidad de los demás (Hemingway, 2012); el diseño de nuestros cerebros para conectar con las experiencias de otros, en un nivel fundamental (Bassett \& Gazzaniga, 2011); la noción de que el procesamiento neurológico es en gran parte inconsciente; la noción de que cada persona es única y tiene su propia perspectiva sobre el mundo (Écija y Velasco, 2014).

Se indagó así, en las conceptualizaciones de aquellas habilidades o competencias que favorecen la interacción entre los individuos y se encontró que, Bar-On et al. (2003) proponen, a partir de los postulados de Salovey y Mayer (1990), el concepto de inteligencia socio-emocional, definida como la capacidad para hacerle seguimiento a los sentimientos propios, diferenciarlos y utilizarlos para actuar y pensar. Bassett y Gazzaniga (2011) permiten pensar este tipo de inteligencia, desde una perspectiva evolutiva, al plantear que la necesidad de conectar con los demás pudo haber comenzado como una estrategia básica de supervivencia. Como miembros de un grupo, los seres humanos podrán ser más fuertes que individualmente (se pueden defender unos a otros ante el peligro, e incluso pueden sacar provecho de los recursos individuales de cada miembro a nivel colectivo en términos de solidaridad, eliminando así la competitividad). Para ellos, un individuo aislado siempre estará en desventaja competitiva frente a un grupo cohesionado, dado que nuestros cerebros están diseñados para conectar, en un nivel muy fundamental, con las experiencias de otros.

A partir de estas definiciones, fue necesario considerar la inteligencia socioemocional, en dos vías de composición, la inteligencia emocional, y la inteligencia social. Se entiende la primera, a partir de la conceptualización de Écija y Velasco (2014) que la proponen como aquella que se focaliza en la perspectiva del "yo" y "mis emociones". Afirma el mismo autor que, al revisar la noción de inteligencia social, el centro de esta reside en la perspectiva del "nosotros" y "nuestras emociones" (Écija y Velasco, 2014). Es decir, que nuestros cerebros están vinculados unos con otros, dado que, somos seres relacionales. Así, la comprensión 
social que se realiza en entornos colectivos requiere inferir estados mentales de quienes participan en estas interacciones y posteriormente, ofrecer posibles soluciones o explicaciones a problemas complejos que conllevan otros problemas en sí.

A partir de esta perspectiva del concepto de inteligencia social, se encontró adicionalmente que, Braslavksy (2004) —basada en los postulados del desarrollo cultural que expone Vigotsky - pone de manifiesto la importancia de los procesos culturales en la diferenciación de la especie humana respecto al resto de animales. Así, "en condiciones apropiadas, el niño primitivo realiza un desarrollo cultural normal y alcanza el nivel intelectual de un hombre culto" (Braslavsky, 2004, p. 30), es decir, en casos ideales, los procesos cognitivos básicos, se desarrollarían en procesos cognitivos superiores, únicamente si hay contextos que lo permitan. La escuela, a través del proceso de educación se convierte entonces, en un potente espacio de culturización e inmersión del sujeto en la moral, en el cuidado de las interacciones con otros y en mantener vínculos que permitan soluciones pacíficas a los dilemas que se presentan cotidianamente (Herrera, 2006).

Es importante mencionar que diferentes autores otorgan relevancia al desarrollo de habilidades que permitan reconocer de forma consciente los sentimientos de los demás, para establecer vínculos, resolver problemas de diversa naturaleza a nivel personal e interpersonal, que se planteen la cotidianidad y la capacidad de automotivarse, mediante la interacción con el entorno y así, generar emociones positivas. Así, la inteligencia social, con base biológica en la cognición social y teoría de la mente y logrando un correcto aprendizaje socio-cultural para el manejo emocional, deben ser equiparados a los procesos cognitivos y no deberían ser trabajados explícitamente, en los diferentes sectores escolares, por adultos mediadores (Tirapú-Ustárroz et al., 2007; García, 2008; Lieberman, 2013; Iacoboni et al., 2005; Rizzolatti \& Craighero, 2004; Butman, 2001; Adolphs 2003; GrandeGarcía, 2009; Frith \& Frith, 2012). Sin embargo, impera la importancia de reconocer cómo se fortalecen estos procesos en escenarios diversos, en este caso, los urbanos y rurales.

Tal como lo exponen Herrera, Buitrago y Cepero (2017), los sectores educativos rurales y urbanos, no sólo en Colombia, sino en Latinoamérica siguen presentando heterogeneidad en el desarrollo de la inteligencia socio-emocional, lo cual para Saadi, Honarmand, Najarian, Ahadi y Askari (2012), representan habilidades constructivas de interacción y deberían pensarse de manera específica para cada contexto.

\section{Diferencias entre los sectores educativos rurales y urbanos: repercusiones en el desarrollo de la inteligencia social}

En Colombia existe diversidad de regiones con particularidades ambientales, geográficas, socioculturales e históricas. Según el Informe Nacional del Desarrollo Humano, el 75,5\% del territorio nacional, es decir, tres cuartas partes del mismo son zonas con baja, media o alta ruralidad presentando menores índices de productividad a nivel económico y mayores índices de violencia, respecto al restante $25 \%$ de zonas urbanas del país (Gaviria, 2017; Figueroa et al., 2018; Morera, Figueroa y Martínez, 2016). Este sector debe enfrentar actualmente desafíos contextuales, tales como una geografía difícil, una población dispersa, el distanciamiento gradual del conflicto armado en una guerra de 52 años (que en muchas regiones sigue estando presente, ya que algunos grupos armados aún no se 
han desmovilizado) y un sistema social, educativo y económico desventajoso, comparado con otras zonas del país.

Según los datos demográficos del Departamento Administrativo Nacional de Estadística (DANE), para el año 2015, aproximadamente 11 millones de personas (cerca del 24\% de la población total colombiana) habitan en las zonas rurales del país, que ocupan más del 80\% del territorio nacional, encontrándose altamente dispersas y separadas de los centros urbanos (MEN, 2017); el 44\% de la población que está en zonas rurales, se encuentra en situación de pobreza multidimensional, en materia de acceso a educación, salud, trabajo y condiciones de habitabilidad (MEN, 2017).

A partir de este panorama, se expone la posibilidad de pensar los sectores urbanos y rurales en Colombia, como facilitadores o limitadores del desarrollo de la inteligencia social, en la medida en que son contextos con particularidades definidas y diferenciadas que estimulan por vías diferentes, los procesos de socialización con el otro.

Sin embargo, ambos sectores pueden representar en su forma, entornos de riesgo psicosocial que amenazan el desarrollo de habilidades sociales sanas, disociando el proceso del desarrollo de la inteligencia social, junto con procesos cognitivos, que deberían darse de forma paralela (Urquijo et al., 2017). Aunque si bien, en términos de desarrollo y de globalización, los entornos urbanos ofrecen ciertas "ventajas" para generar procesos más amplios de vinculación — mayores tasas de habitantes, acceso inmediato a redes de comunicación, grupos sociales, etc.

Pensando la educación como un espacio de construcción de redes de sanas interacciones sociales, se encuentra que, los tiempos en que niños, niñas y adolescentes participan de los mismos son diferentes en función de las zonas en donde crecen. Así y según los datos del Departamento Nacional de Planeación (DNP) (citado por MEN, 2017), para el 2015 la población que habita en zonas rurales lograba cumplir 5,7 años de escolaridad, en contraste con quienes se encuentran en zonas urbanas, con un aproximado de 9,5 años en la escuela. Para el año siguiente, la cifra varió en zonas rurales con un aumento a 6,0 años y en zonas urbanas 9,6. El MEN cita además que, la diferencia neta en la cobertura de la educación secundaria y media entre ambos sectores es de aproximadamente 20 puntos porcentuales. Además, un estimado del $50 \%$ de las instituciones educativas rurales tienen un desempeño inferior y bajo, respecto a un $20 \%$ en los establecimientos urbanos.

Sin embargo, no es propósito de este apartado mantener la discusión entre las ventajas o desventajas que tenga un sector sobre el otro, sí corresponde plantear la posibilidad de pensar en los escenarios educativos, tanto urbanos como rurales como un factor protector que permitan el aprendizaje de elementos puntuales, particulares, contextuales y explícitos para dimensionar no sólo cómo se tejen las relaciones sociales, sino para comprender cómo se interactúa en las mismas. Lo anterior supone repensar tanto en el sector educativo urbano como rural, la educación para ambos sectores, la estructura rígida del desarrollo de la inteligencia cognitiva, a través de conocimientos de peso científico y exacto, e intentar el desarrollo de la inteligencia socio-emocional que, actualmente son reducidos (Maree, 2001). La necesidad de permanecer vinculado al otro y percibir su aceptación, motiva, mantiene y fortalece las interacciones con personas muy cercanas, amigos, recién conocidos, e incluso, con personas desconocidas, indiferenciadamente entre 
sectores urbanos y rurales, siendo un hecho ineludible en la condición humana, que debe ser desarrollada y fomentada, especialmente en la escuela.

Habría que valerse del concepto de la cognición social, o teoría de la mente, que ha sido fuertemente abordado para comprender la base neurocognitiva de la IS y así, apuntar hacia la implementación de estrategias que tienen como principio fundamental, reconocer la humanidad de los demás (Hemingway, 2012; Restrepo, Vásquez y Ortiz, 2018), a través de espacios de aprendizaje de las interacciones sociales que suceden en cada contexto, siendo necesario, incluso, develar constantemente las tensiones que ocurren en el currículo oculto entre docentes y estudiantes.

Autores como Mejía (2011), Herrera, Buitrago y Cepero (2017) y Nivagara (2018) consideran que, la tendencia actual de intervención educativa está dirigida a responder a las necesidades globales en sus contenidos de conocimiento, dando lugar a la creación de currículos formales que apuntan a resolver problemáticas que se encuentran, principalmente en el sector urbano. El abordaje teórico-científico en la creación de temáticas de intervención y su necesaria inversión en la formación de estos asuntos, como la resolución de conflictos interpersonales, suele pensarse con mayor frecuencia para los sectores urbanos, donde hay mayor retorno en la distribución de recursos económicos; materiales de apoyo didáctico y formativos; rutas de acceso e infraestructura adecuada, lo que representa una desventaja para el aprovechamiento de los espacios en las escuelas rurales, la aprehensión de los contenidos formales, y el aprendizaje de normas, valores y principios de interacción social (Cossio, 2014).

Así, la brecha sigue abriéndose, incluso para asumir criterios de evaluación del desempeño de niñas, niños y adolescentes en las escuelas rurales. Los estándares pueden llegar a ser precisos a la hora de ser formulados, pero a la hora de aplicarlos, carecen de aplicación. Apoyando esta perspectiva, Echavarría, Vanegas, González y Bernal (2019) plantean que el saber más cercano que tiene la población infantil en las zonas rurales "son expertos en las labores del hato, saben arriar, enlazar, cultivar, cocinar, pero estudian poco. Razón por la cual, podría inferirse que no obtienen los mejores resultados en las pruebas estandarizadas" (p. 22).

En la página web de Empresarios por la Educación (Hernández, 2018) se encuentra la siguiente afirmación:

La combinación entre deserción escolar y servicios básicos insatisfechos puede ser una de las causas por las que a los jóvenes pobres y rurales les va tan mal en las pruebas Pisa. La Fundación Empresarios por la Paz informó que a pesar de que Colombia fue el país con mayores mejoras en este examen entre 2006 y 2015, frente a sus pares latinoamericanos, también fue el que menos disminuyó las brechas entre los niveles socioeconómicos más altos y los más bajos (párr. 7).

Con esta información, cabe problematizar entonces, sobre el lugar de la mediación que tiene la escuela sobre conductas socialmente dirigidas (como una precondición para el aprendizaje cognitivo y social) que integren y desarrollen las dinámicas de la interacción social, incluyendo la confrontación de estereotipos, prejuicios, creencias, valores, normas y actitudes frente al otro, para dimensionarlo como un ser con deseos, pensamientos, sentimientos, ansiedades, y así, favorecer la comprensión y la correspondencia en las relaciones interpersonales. 
Frente a esto, Grande-García (2009) y Andrés (2005) ya habían argüido desde una perspectiva histórico cultural que, estas interacciones deben estar mediadas por el incentivo en los niños y niñas de la expresión constante de sus pensamientos y emociones, fortaleciendo una estructura que, además, les permita contener y mediar con el ímpetu de estas expresiones, en relación con los demás, como lo reafirma Mejía (2004) y (Koplow, 2005). Sin embargo, queda el vacío en quienes enseñan en las escuelas rurales más alejadas de los cascos urbanos, ya que como lo plantean Diniz y Lima (2015), los maestros en el campo viven todo tipo de vicisitudes y se enfrentan a políticas homogéneas, abstractas y sin suficiente lectura analítica de las necesidades que enfrenta la escuela rural con aulas multigrados, dentro de una ruralidad dispersa.

\section{Inclusión de la inteligencia social en los diseños curriculares}

Los diseños curriculares evidencian la utilidad que tienen como medio para esquematizar y representar modelos específicos de cómo funciona la realidad en torno, en este caso puntual, al desarrollo de la inteligencia social y la forma de transmitirlo en las aulas de clase, para fomentar una constante experiencia de convivencia pacífica (Zamudio y Martínez, 2017).

La educación y el currículo, si bien tienen distinciones específicas, no pueden considerarse por separado. Si la intención de la educación radica en preparar a los Seres Humanos, para estar en sociedad, debe haber un currículo que logre poner de manifiesto el complejo sistema de intercambios que suceden dentro del aula de clase, y que, a partir de la comprensión de este fenómeno, pueda extrapolarse hacia los diferentes ámbitos en los que se desarrolla la vida cotidiana de los sujetos (Díaz, 2006; Ratto, 2015).

Para mediar entre el mundo de las intenciones y el de las actividades concretas, en el entorno escolar, existe un mecanismo posibilitador de modelos estructurados que llevan la realidad del entorno a las aulas de clase, tal como lo sugiere Ratto (2015), al citar a Pérez, hablando del diseño curricular. En el mismo sentido Díaz (1993) afirma que actualmente el desarrollo curricular propende por darle el lugar al docente como un mediador del aprendizaje y de facilitador de las interacciones que se tejen entre los estudiantes.

Si bien, es a través de la participación de los niños y niñas en la vida escolar, en donde se adquieren diversos contenidos a nivel teórico que permiten un mayor afianzamiento de los mismos en la vida real, es imperante mencionar que, también se han pensado el currículo, como un dispositivo pedagógico para la construcción del conocimiento y puesta en acción, donde, además, se aprendan actitudes, conductas y prácticas sociales, en función de construir la identidad de los estudiantes, como individuos y como actores sociales.

Por esto, debe encontrarse el reflejo de los intereses de los alumnos en la cultura escolar, para evitar fracturas a nivel de los sistemas que, conlleven al deseo de desertar y rechazar los ambientes educativos (Sacristán, 2010). Según planteaba Koplow (2005), se deben generar ambientes que sean emocionalmente seguros para que los niños y niñas lleguen a ser atentos y productivos, pero sobretodo, socialmente exitosos.

Actualmente, en las escuelas, tanto rurales como urbanas, se ha pasado de la violencia física a la violencia emocional, moral y simbólica, lo que complejiza el fortalecimiento de las relaciones interpersonales (Romero, 2011; Gil, 2008). A decir de Mena y Huneeus (2017), 
para lograr una sana convivencia escolar deben definirse estrategias para minimizar focos de violencia y conductas disruptivas. No basta con entender las bases biológicas del cerebro, en función de las relaciones sociales, o cómo se estructuran, desde una perspectiva psicosocial, es necesario trabajar de forma explícita y en función del aprendizaje, la relación que tiene la adaptación que permite nuestro sistema nervioso al medio que nos rodea y a la superación de las diversas problemáticas que generan las interacciones sociales a diario (Ortega, s.f.).

Así, el currículo puede entenderse en tres lógicas de acción: el currículo formal, el currículo real y el currículo oculto. El currículo formal planifica los programas académicos, según demandas universales, que son particularizadas en cada aula de clase, a través del currículo real. Sin embargo, existen aprendizajes implícitos y tensiones en medio del proceso de planeación, desarrollo y evaluación curricular que también es fuente de transmisión de aprendizaje, y es el currículo oculto (Ratto, 2015; Devís, Fuentes \& Sparkes, 2005; Díaz, 1993).

Es decir, tanto explícita como implícitamente, la escuela contribuye constantemente a la consolidación de las identidades de los niños, niñas y adolescentes a nivel personal y social. Se convierte el aula de clase en un contexto de socialización, donde surgen experiencias altamente significativas en la consolidación del sujeto consigo mismo y con su entorno, generando profundas raíces en el presente y brindando un lugar del individuo, en el mundo. Así lo expresa Sacristán (2001) en su libro "Educar y convivir en la cultura global".

Más adelante, Devís et al. (2005) manifiestan la importancia de la formación del profesorado como facilitadores de espacios de construcción de tejido social. También LedesmaAyora (2014) retoma los postulados de Vigotsky, para mencionar cómo la mediación del docente, permitirá construir en conjunto con los estudiantes el desarrollo de habilidades sociales, dentro del enfoque histórico-cultural, en búsqueda del fortalecimiento de la Inteligencia social, como habilidad para enfrentar las diferentes situaciones a nivel plural, a partir del desarrollo de la vida emocional.

En otras palabras, los docentes — desde este punto de vista - son mediadores en la construcción de recursos internos para que los niños, niñas y adolescentes soporten las hostilidades del medio. En palabras de Goleman (2006): "dado el modo en que nuestro cerebro aprende la capacidad de recuperación social, (...) si un niño se ofusca, el valor de la Inteligencia social emerge cuando éste logra cierto control sobre su propia reacción" (p. 263), así, los niños y niñas requieren un entrenamiento para enfrentarse a las variaciones de la vida social.

Se plantea entonces, adentrarse en los espacios del currículo, ya que la mayoría de las situaciones problemáticas a nivel escolar, están relacionadas con este. El fracaso escolar, la forma en que los docentes se relacionan con los alumnos, las normas puestas en clase, etc., hacen parte de los contenidos pedagógicos que se les ofrece a los estudiantes y aún más, con la manera en que se les ofrecen.

Se han documentado varios procesos en los que se ha propuesto evaluar y fortalecer la inteligencia social en contextos educativos. Es el caso de la Universidad del Sur de Australia, donde Nunan, George y McCausland (2000) proponen repensarse las formas en que se soportan los procesos de enseñanza y aprendizaje, capacitando a los docentes 
en generar mejores vínculos sociales que facilite a los estudiantes una mejor aprehensión de los contenidos vistos en clase.

También, se encontró que en la Harvard Business School de la Universidad de Harvard, Hamel y Prahalad (1990) concibieron un programa de entrenamiento en 'competencias centrales' donde los gerentes y líderes de las compañías, realizarían sus procesos de capacitación y formación, desde el manejo emocional; luego, en la misma Universidad, Jaeger (2003) menciona la importancia del desarrollo de la inteligencia emocional en los egresados de su programa formativo.

La Universidad de Pretoria, en Sudáfrica, realizó un programa de acompañamiento y patrocinio de población vulnerable por la pobreza, para realizar estudios universitarios; realizaron un estudio para evaluar el desarrollo de la Inteligencia social-emocional en medio de un proceso formativo longitudinal (Maree, 2011).

En España, específicamente en la Universidad de la Rioja, Martínez-Pinero (2014), desarrollaron un programa para capacitar en inteligencia social a los docentes de primeros ciclos educativos. A nivel latinoamericano, la Universidad Nacional de Trujillo, en Perú, también planteó un modelo para el desarrollo de la inteligencia social en estudiantes de bachillerato, basado en las teorías de los procesos cognitivos de Luria, la teoría psicopedagógica socio cultural de Vigotsky (Braslavsky, 2004), la teoría de la pedagogía conceptual de Zubiría (2006), la teoría de la Inteligencia social de Goleman (2006) y la teoría de Carlos Álvarez de Zayas (Zúñiga y Ortiz, 2017).

En Colombia, la Universidad de Antioquia en el 2003, acompañó un trabajo de grado para la licenciatura en educación especial, con el diseño de una propuesta pedagógico-didáctica basada en sistemas alternativos de comunicación, para la enseñanza y el aprendizaje de la inteligencia social y emocional en niños con parálisis cerebral (Betancur, Montoya y Pineda, 2003).

La Universidad de San Buenaventura en Bogotá, según Romero (2008), ha propuesto un programa de estrategias y herramientas para el desarrollo de la inteligencia social, a partir de los ciclos educativos propuestos por la pedagogía de la humanización que, según ellos, permitiría el aprendizaje de competencias ciudadanas, responsabilidad social, capacidades de valoración, respeto, negociación y concertación.

\section{Conclusiones}

Se evidencian notables diferencias entre la educación rural y urbana, dado que los diseños curriculares no están siendo contextualizados al contexto real educativo y social, sino que tienden a ser estandarizados en sus contenidos, estrategias pedagógicas y modelos de aprendizaje, que parecen dar mejor respuesta o estar pensados en mayor proporción para entornos urbanos.

Lo anterior supone la necesidad política de repensar los escenarios educativos acorde a las características del contexto, para determinar las formas de intervención educativa que den respuesta oportuna a las necesidades y expectativas de la población a que se dirigen los esfuerzos de enseñanza-aprendizaje. Aunado a considerar que el currículo más allá de brindar una formación académica, debe dar respuesta al desarrollo integral y personal de los estudiantes que se forman, de esta manera recobra importancia el desarrollo de 
la inteligencia social en el entorno educativo; se favorece la flexibilidad curricular como respuesta a las particularidades presentes en los procesos de aprendizaje individual y colectivo.

Finalmente, pensar la educación desde un enfoque distinto que integra las lecturas neuro-cognitivas; psicológicas (educativa y social), y pedagógicas de las habilidades de socialización, favorece la construcción de diseños curriculares contextualizados que integran metodologías diversas como aporte al desarrollo de procesos educativos integrales en el sector educativo, como lo son la convivencia escolar, la formación de competencias ciudadanas y la construcción de paz.

\section{REFERENCIAS}

Adolphs, R. (2003). Cognitive neuroscience of human social behaviour. Nature Reviews, 4(3), 165-178. https://doi.org/10.1038/nrn1056

Albrecht, K. (2006). Inteligencia social. Barcelona: Vergara.

Andrés, C. (2005). La educación emocional en edades tempranas y el interés de su aplicación en la escuela: programas de educación emocional, nuevo reto en la formación de los profesores. Tendencias Pedagógicas, 10, 1-17. Disponible en https://revistas.uam. es/tendenciaspedagogicas/article/view/1856

Ardila, R. (2011). Inteligencia . ¿Qué sabemos y qué nos falta por investigar? Revista de la Academia Colombiana de Ciencias Exactas, Físicas y Naturales, 35(134), 97-103. Disponible en https://biblat.unam.mx/es/revista/revista-de-la-academia-colombianade-ciencias-exactas-fisicas-y-naturales/articulo/inteligencia-que-sabemos-y-que-nosfalta-por-investigar

Bar-On, R., Tranel, D., Denburg, N. L. y Bechara, A. (2003). Exploring the neurological substrate of emotional and social intelligence. Brain, 126(8), 1790-1800. https://doi. org/10.1093/brain/awg177

Bassett, D. S. \& Gazzaniga, M. S. (2011). Understanding complexity in the human brain. Trends in cognitive sciences, 15(5), 200-209. https://doi.org/10.1016/j.tics.2011.03.006

Betancur, A., Montoya, D. y Pineda, M. (2003). Propuesta pedagógico-didáctica basada en sistemas alternativos de comunicación para la enseñanza y el aprendizaje de la inteligencia social y emocional en niños con parálisis cerebral. [Tesis grado]. Universidad de Antioquia, Medellín, Colombia.

Braslavsky, B. (2004). ¿Primeras letras o primeras lecturas? Buenos Aires: Fondo de Cultura Económica. Recuperado de http://www.ispgposadas.edu.ar/files/BERTA\%20 BRASLAVSKY\%20\%20P-1-Compr.pdf

Butman, J. (2001). La cognición social y la corteza cerebral. Revista Neurológica Argentina, 26(3), 117-122. Disponible en http://www.contextos-revista.com.co/Revista\%204/ A5_Una\%20introduccion\%20a\%20la\%20cognicion20social.pdf 
Calle, D. (2014). Cerebro y cognición social. Un puente entre la neurociencia y la construcción social del sujeto. Realitas, Revista de ciencias sociales, humanas y artes, 2(1), 51-56. Disponible en https://revista-realitas.webnode.com.co/

Corvalán, J. (2006). Educación para la población rural en siete países de América Latina. Síntesis y análisis global de resultados por países. Revista Colombiana de Educación, 51, 40-79. https://doi.org/10.17227/01203916.7684

Cossio, J. A. (2014). Pedagogía y calidad de la educación: una mirada a la formación del maestro rural. Sophia, 10(1), 14-23. Disponible en https://revistas.ugca.edu.co/index. $\mathrm{php} /$ sophia/article/view/214

Creswell, J. W. (1998). Qualitative Inquiry and Research Design: Choosing Among Five Traditions. Thousand Oaks: Sage Publications.

De Zubiría, J. (2006). Los modelos pedagógicos: hacia una pedagogía dialogante. Bogotá, D.C.: Coop. Editorial Magisterio.

Devís, J., Fuentes, J. y Sparkes, A. (2005). ¿Qué permanece oculto del currículum oculto? Las identidades de género y de sexualidad en la educación física. Revista Iberoamericana de Educación, 39(1), 73-90. Recuperado de https:/rieoei.org/historico/ documentos/rie39a03.pdf

Díaz, Á. (2006). La educación en valores: Avatares del currículum formal, oculto y los temas transversales. Revista electrónica de investigación educativa, 8(1), 1-15. Disponible en https://redie.uabc.mx/redie/article/view/117

Díaz, B. F. (1993). Aproximaciones metodológicas al diseño curricular hacia una propuesta integral. Tecnología y Comunicación Educativas, (21), 19-39. Recuperado de http://tyce.ilce.edu.mx/tyce/21/TecyComEduNo21_A02.pdf

Diniz, M. \& Lima, J. R. (2015). Gênero e Docência: Olhares sobre a formação de professoras rurais no Brasil e na Colômbia. Revista Historia de la Educacion Latinoamericana, 17(24), 167-190. https://doi.org/10.19053/01227238.3305

Echavarría, C., Vanegas, J. H., González, L. y Bernal, J. S. (2019). La educación rural "no es un concepto urbano". Revista de la Universidad de La Salle, (79), 15-40. https://doi.org/10.19052/ruls.vol1.iss79.2

Écija, C. y Velasco, L. (2014). Inteligencia social: aplicación práctica en el contexto educativo. La humanización de las relaciones sociales. Madrid: Ministerio de Educación, Cultura y Deporte. Disponible en https://sede.educacion.gob.es/publiventa/inteligencia-social-aplicacion-practica-en-el-contexto-educativo-la-humanizacion-de-las-relaciones-sociales/ensenanza-sociologia-convivencia/16528

Espejo, E. (2016). Necesidad de una práctica pedagógica de aula que dé respuesta a la diversidad. Paideia, (59), 85-98. Disponible en http://revistasacademicas.udec.cl/index. php/paideia/article/view/694

Figueroa-Torres, Y. N., Pérez-Bella, S. M., Villamarín-Monroy, C. L. y Rincón-Pérez, A. (2018). Antecedentes, realidades y retos de los programas nacionales para 
la Reforma Rural Integral. Ciencia y Agricultura, 15(1), 101-110. https://doi. org/10.19053/01228420.v15.n1.2018.7761

Frith, C. \& Frith, U. (2012). Mechanisms of Social Cognition. Annual Review in Psychology, 63(1), 287-313. https://doi.org/10.1146/annurev-psych-120710-100449

García, E. (2008). Neuropsicología y educación. De las neuronas espejo a la teoría de la mente. Revista de psicología y educación, 1(3), 69-90. Recuperado de http://www. revistadepsicologiayeducacion.es/pdf/27.pdf

Gaviria, J. A. (2017). Problemas y retos de la educación rural colombiana. Educación y Ciudad, (33), 53-62. https://doi.org/10.36737/01230425.v0.n33.2017.1647

Gil, M. (2008). Convivir en la diversidad, una propuesta de integración social desde la escuela. Bogotá, D.C.: Magisterio.

Goleman, D. (2006). Inteligencia social, la nueva ciencia para mejorar las relaciones humanas. México, D.F.: Planeta.

Grande-García, I. (2009). Neurociencia social: El maridaje entre la psicología social y las neurociencias cognitivas. Revisión e introducción a una nueva disciplina. Anales de psicología, 25(1), 1-20. Disponible en https://revistas.um.es/analesps/article/ view/71441

Guzmán, K., Fernández, M. y Villalba, L. (2016). Emociones en niños, niñas y adolescentes desde la experiencia del desplazamiento y la vinculación a los grupos armados en Colombia. Panorama, 10(19), 85-96. https://doi.org/10.15765/pnrm.v10i19.834

Hamel, G. \& Prahalad, C. K. (1990). The core competence of the corporation. Harvard business review, 90(3), 79-91. Disponible en https://hbr.org/1990/05/the-core-competence-of-the-corporation

Hemingway, A. (sept. 28, 2012). Humanity, respect and dignity are the foundations of practice. Nursing times, 108(40), 11-11. Available: https://www.nursingtimes.net/ roles/nurse-managers/humanity-respect-and-dignity-are-the-foundations-of-practice-28-09-2012/

Hernández, J. (mayo 19, 2018). La difícil situación de las escuelas rurales en Colombia. El Espectador. Disponible en https://www.elespectador.com/colombia2020/territorio/ la-dificil-situacion-de-las-escuelas-rurales-en-colombia-articulo-856698/

Hernández, R., Fernández, C. y Baptista, P. (2014). Metodología de la Investigación. México, D.F.: McGraw-Hill.

Herrera, L. (2006). La educación y la cultura: una lectura y propuesta desde la filosofía de la praxis. Sophía, 1(1), 187-231. https://doi.org/10.17163/soph.n28

Herrera, L., Buitrago, R. E. y Cepero, S. (2017). Inteligencia emocional en ninos colombianos de educacion primaria. Analisis por contexto y genero. Universitas Psychologica, 16(3), 1-10. https://doi.org/10.11144/Javeriana.upsy16-3.eips 
Hincapie, G., Montoya, I. y Bustamante, J. (2017). Sistema vial terciario y educación rural en Antioquia (Colombia): un modelo probit ordenado y multivariado. Ecos de Economía, 21(44), 72-85. https://dx.doi.org/10.17230/ecos.2017.44.3

Iacoboni, M., Molnar-Szakacs, I., Gallese, V., Buccino, G., Mazziotta, J. C. \& Rizzolatti, G. (2005). Grasping the intentions of others with one's own mirror neuron system. PLoS biology, 3(3), 529-535. Available: https://journals.plos.org/plosbiology/article/ file?type=printable\&id=10.1371/journal.pbio.0030079

Jaeger, A. (2003). Job competencies and the curriculum: An inquiry into emotional intelligence in graduate professional education. Research in Higher Education, 44(6), 615-639. https://doi.org/10.1023/A:1026119724265

Koplow, L. (2005). Escuelas que curan: la construcción de climas emocionales saludables. Buenos Aires: Troquel.

Ledesma-Ayora, M. (2014). Análisis de la teoría de Vygotsky para la reconstrucción de la inteligencia social. Cuenca: Universidad Católica de Cuenca. Disponible en http://hdl. handle.net/10366/127738

León, D. y Cárdenas, F. P. (2016). Aproximación Neurodinámica a la Cognición Social. Universitas Psychologica, 15(5), 1-25. http://dx.doi.org/10.11144/Javeriana.upsy15-5. ancs

Lieberman, M. D. (2013). Social: Why our brains are wired to connect. Oxford: OUP.

López, L. R. (2018). Ruralidad y educación rural. Referentes para un Programa de Educación Rural en la Universidad Pedagógica Nacional. Revista Colombiana de Educación, (51), 138-159. https://doi.org/10.17227/01203916.7687

Manes, F. y Niro, M. (2014). Usar el cerebro. (4 ed.). Buenos Aires: Planeta.

Maree, K. (2011). El Proyecto Limpopo: evidencia empírica sobre el concepto de inteligencia emocional-social. Revista Electrónica Interuniversitaria de Formación del Profesorado, 14(3), 107-129. Disponible en https://revistas.um.es/reifop/issue/view/11921

Martínez-Pineiro, B. (2014). El desarrollo de la Inteligencia social y emocional en Educación Infantil. [Trabajo de grado]. Universidad Internacional de La Rioja, Barcelona, España. Disponible en https://reunir.unir.net/handle/123456789/2300

Mejía, M. (2011). Educaciones y pedagogías críticas desde el sur. La Paz: Ministerio de Educación. Recuperado de https://www.minedu.gob.bo/files/publicaciones/veaye/ EDUCACIONES-Y-PEDAGOCIAS-ed.-Bolivia.pdf

Mejía, A. (2004). Competencias ciudadanas: de los estándares al aula. Revista de Estudios Sociales, 19, 127-128. Disponible en https://journals.openedition.org/revestudsoc/24449

Mena, M. \& Huneeus, M. (2017). Convivencia Escolar para el aprendizaje y buen trato de todos: Hacia una mejor comprensión del concepto. Cultura Educación y Sociedad, 8(2), 9-20. https://doi.org/10.17981/cultedusoc.8.2.2017.01 
Morera, W., Figueroa, A. G. y Martínez, A. (2016). Currículo en los aprendizajes de los niños de la escuela rural: Garantía al Derecho a la Educación. Via Inveniendi Et Iudicandi, 11(2), 181-194. https://doi.org/10.15332/s1909-0528.2016.0002.08

Nivagara, D. (2018). O currículo local como política para a preservação e/ou transformação da cultura rural (do campo). Amazônica, 21(1), 302-320. Disponible en https:// sinchi.org.co/revista

Nunan, T., George, R. \& McCausland, H. (2000). Rethinking the ways in which teaching and learning are supported: The Flexible Learning Centre at the University of South Australia. Journal of Higher Education Policy and Management, 22(1), 85-98. https://doi.org/10.1080/713678130

Ortega, J. (s.f.). Neuropsicopedagogía del aprendizaje y la conducta. [Contenido temático]. Girona: UNED. Recuperado de https://extension.uned.es/archivos_publicos/webex_actividades/4366/programaneuropsicopedagogiaaprendizajeconducta.pdf

Papalia, D. y Martorell, G. (2017). Desarrollo Humano. México, D.F.: Mc Graw Hill.

Preal. (1998). El futuro está en Juego. [Informe] Santiago de Chile: Preal.

Ratto, M. C. (2015). Teoría y diseño curricular. México, D.F.: Trillas.

República de Colombia. Congreso de la República. (8 de febrero de 1994). Ley general de educación. [Ley 115]. DO: 41.214. Recuperado de http://www.mineducacion.gov. co/1621/articles-85906_archivo_pdf.pdf

República de Colombia. MEN. (2017). Plan especial de educación rural - Hacia el desarrollo rural y la construcción de paz. [Online]. Recuperado de https://www.mineducacion.gov.co/1759/articles-385568_recurso_1.pdf

República de Colombia. MEN. (2013). Ley de convivencia escolar. [Ley 1620]. DO: 48.733. Recuperado de https://www.mineducacion.gov.co/1759/articles-327397_archivo_pdf_ proyecto_decreto.pdf

República de Colombia. MEN. (septiembre 11 de 2013). Reglamentación Ley 1620 de 2013, que crea el Sistema Nacional de Convivencia Escolar y Formación para el Ejercicio de los Derechos Humanos, la Educación para la Sexualidad y la Prevención y Mitigación de la Violencia Escolar. [Decreto 1965]. DO: 48.910.

República de Colombia. MinSalud. (2012). Plan de acción de salud: Primero 1,000 días de vida. [Online]. Recuperado de https://www.minsalud.gov.co/sites/rid/Lists/BibliotecaDigital/RIDE/VS/PP/ENT/planeacion-primeros-mil-dias.pdf

Restrepo, Y., Vásquez, C. y Ortiz, L. (2018). La cognición social: abriendo puertas para la convivencia escolar. En, R. Jaramillo. (Ed). Formación y Convivencia (pp. 23-33). Medellín: Rodrigo Jaramillo.

Rivas, A. (2015). América Latina después de PISA: Lecciones aprendidas de la educación en siete países (2000-2015). Buenos Aires: CIPPEC. Disponible en https://www. cippec.org/publicacion/america-latina-despues-de-pisa-lecciones-aprendidas-de-laeducacion-en-siete-paises-2000-2015/ 
Rizzolatti, G. \& Craighero, L. (2004). The mirror-neuron system. Annual Review of Neuroscience, 27(1), 169-192. https://doi.org/10.1146/annurev.neuro.27.070203.144230

Rizzolati, G. Fadiga, L., Gallese, V. \& Fogassi, L. (1996). Premotor cortex and the recognition of motor actions. Cognitive \& Brain Research, 3(2), 131-141. https://doi. org/10.1016/0926-6410(95)00038-0

Rodelo, M. y Castro, S. (2016). Reflexiones sobre la educabilidad: elemento fundamental en el proceso educacional del desarrollo humano. Cultura Educación y Sociedad, 7(2), 94-104. Disponible en https://revistascientificas.cuc.edu.co/culturaeducacionysocie$\mathrm{dad} /$ article/view/1104

Romero, P. (2011). Estrategias y herraminetas para la inteligencia social. Una interpretación desde los ciclos educativos. Itinerario Educativo, 25(58), 199-225. https://doi. org/10.21500/01212753.1455

Romero, P. (2008). Pedagogía de la Humanización en la educación inicial. [Informe avances investigación]. Bogotá, D.C.: Universidad San Buenaventura. Recuperado de http://biblioteca.usbbog.edu.co:8080/Biblioteca/BDigital/36711-01.pdf

Ruíz-Ruíz, J. C., García-Ferrer, S. y Fuentes-Durá, I. (2006). La relevancia de la cognición social en la esquizofrenia. Apuntes de Psicología Colegio Oficial de Psicología, 24(1), 137-155. Disponible en http:/www.apuntesdepsicologia.es/index.php/revista/ article/view/74/76

Saadi, Z. E., Honarmand, M. M., Najarian, B., Ahadi, H. \& Askari, P. (2012). Evaluation of the effect of emotional intelligence training on reducing aggression in second year high school female students. Journal of American Science, 8(5), 209-212. Available: http://www.sciencepub.net/american/am0805/026_9071am0805_209_212.pdf

Sacco, D. F., Merold, S. J., Lui, J. H., Lustgraaf, C. J. \& Barry, C. T. (2016). Social and emotional intelligence moderate the relationship between psychopathy traits and social perception. Personality and Individual Differences, 95, 95-104. https://doi. org/10.1016/j.paid.2016.02.031

Sacristán, J. G. (2010). Saberes e incertidumbres sobre el curriculum. Valencia: Morata.

Sacristán, G. (2001). Educar y convivir en la cultura global. Madrid: Morata.

Salovey, P. \& Mayer, J. D. (1990). Emotional intelligence. Imagination, cognition and personality, 9(3), 185-211. https://doi.org/10.2190/DUGG-P24E-52WK-6CDG

Soon, C. S., Brass, M., Heinze, H.-J. \& Haynes, J.-D. (2008). Unconscious determinants of free decisions in the human brain. Nature Neuroscience, 11(5), 543-545. https:// doi.org/10.1038/nn.2112

Sternberg, R. J., Forsythe, G. B., Hedlund, J., Wagner, R. K., Williams, W. M., Horvath, J. A. \& Grigorenko, E. (2000). Practical intelligence in everyday life. Cambridge: Cambridge University Press.

Tikka, A. (2006). Social intelligence: A learnable skill or a component of general intelligence. Psychology, 41, 361-368. 
Tirapú-Ustárroz, J., Pérez-Sayes, G., Erekatxo-Bilbao, M. y Pelegrín-Valero, C. (2007). ¿Qué es la teoría de la mente? Revista de Neurología, 44(8), 479-489. https://doi. org/10.33588/rn.4408.2006295

Urquijo, M., Zapata, L., Lewis, S., Pineda-Alhucema, W., Falquez, L. y Lopera-Pérez, D. (2017). Influencia del riesgo social en la teoría de la mente y funciones ejecutivas de adolescentes colombianos. Universitas Psychologica, 16(2), 1-14. https://doi. org/10.11144/Javeriana.upsy16-2.irst

Vélez, O. y Galeano, M. (2002). Investigación cualitativa estado del arte. Medellín: Universidad de Antioquia.

Villamizar, G. y Donoso, R. (2013). Definiciones y teorías sobre inteligencia. Revisión histórica. Psicogente, 16(30), 407-423. Disponible en http://revistas.unisimon.edu.co/ index.php/psicogente/article/view/1927

Weis, S. \& Conzelmann, K. (2015). Social intelligence and competencies. In: International Encyclopedia of the Scocial \& Behavioral Sciences (2 ed.), (pp. 371-379). Oxford: Elsevier. https://doi.org/10.1016/B978-0-08-097086-8.25094-0

Zamudio, L. y Martínez, I. C. (2017). Hacia el fortalecimiento de la atención a la diversidad en Colombia: La educación inclusiva desde la formación de docentes. Paideia, (60), 41-63. Disponible en https://revistas.udec.cl/index.php/paideia/article/view/700

Zúñiga, L. B. y Ortiz, C. L. (2017). Modelo de Inteligencia social para la formación integral de los estudiantes de economía en la Universidad Nacional de Trujillo. Revista Ciencia y Tecnología, 12(4), 113-127. Disponible en http://revistas.unitru.edu.pe/index.php/PGM/article/view/1519

Juan Camilo Lotero Franco es Psicólogo de la Fundación Universitaria Luis Amigo (Colombia). Magíster en neuropsicopedagogía de la Fundación Universitaria Luis Amigo (Colombia). https://orcid.org/0000-0001-9000-5953

Yusty Carolina Restrepo Segura es Psicóloga de la Universidad CES (Colombia). Magister en Intervenciones Psicosociales de la Fundación Universitaria Luis Amigo (Colombia). https://orcid.org/0000-0003-2565-0416

Leisy Magdali Arroyave Taborda es Licenciada en Educación Básica con énfasis en Matemáticas de la Fundación Universitaria Luis Amigo (Colombia). Maestría en Adicciones de la Fundación Universitaria Luis Amigo (Colombia). https://orcid.org/00000003-3982-2805 